\title{
Intracardiac Lethal Injection Euthanasia
}

National Cancer Institute

\section{Source}

National Cancer Institute. Intracardiac Lethal Injection Euthanasia. NCI Thesaurus. Code C116227.

A method of euthanasia whereby a subject is given a lethal intracardiac injection to induce death. 